\title{
A wireless power method for deeply implanted biomedical devices via capacitively-coupled conductive power transfer
}

\author{
Reza Sedehi, Student Member, IEEE, David Budgett, Senior Member, IEEE, Jiang Jincheng, Xia Ziyi, Xin Dai, \\ Member, IEEE, Aiguo Patrick Hu, Senior Member, IEEE, and Daniel McCormick, Member, IEEE
}

\begin{abstract}
Deeply implanted biomedical devices (DIBDs) are a challenging application of wireless power transfer because of the requirement for miniaturisation whilst minimising patient exposure to tissue heating. This work proposes a capacitively-coupled conductive power transfer method for DIBDs, which allows for the safe transfer of power into the body whilst using minimum implant volume. The method uses parallel insulated capacitive electrodes to couple uniform current flow into the tissue and implants. Analytical analyses are presented, which result in a two-port network that describes circuit operation. The two-port network is further simplified for typical DIBD applications where coupling to the external electrodes is low. This results in a simple circuit model of power transfer for which the parameters are easily obtained by experimental measurements. The proposed circuit model has been validated using circuit coupled finite element analysis (COMSOL) and benchtop experiments using a tissue phantom. In addition, the safety aspect of the method has been evaluated via COMSOL simulation of the specific absorption rate (SAR) for various implanted receiver dimensions and implantation depths. Finally, a completed power supply, unaffected by the implantation depth, running at $6.78 \mathrm{MHz}$, delivering $10 \mathrm{~mW}$ deep into the body whilst meeting the IEEE C95.1 basic restriction is presented.
\end{abstract}

\section{INTRODUCTION}

Active biomedical implants require power to operate. The power requirement of such devices, ranging from microwatts to tens of watts [1], can either be supplied by implanted energy storage units, via percutaneous (through the skin) drive lines, or wireless power transfer. Due to the short lifetime, the size of storage units and infection potential of percutaneous drive lines, wireless power transfer (WPT) is becoming the preferred long term power source for implantable devices [2][3][4][5][6]. The most commonly used type of transcutaneous WPT is inductive power transfer (IPT). Inductive power uses a magnetic field to couple an external and an implanted coil to deliver power. However, the implanted coils are difficult to integrate into miniaturised implants due to the presence of metallic components such as the battery and hermetic packaging, which create parasitic loads that may reduce performance. Moreover, miniaturisation is accompanied by higher power densities, leading to high field strengths and circulating currents which make it difficult to meet specific absorption rate limits (SAR) [7].

Ultrasonic, capacitive and galvanic power transfer are alternative technologies which could power highly miniaturised devices. Ultrasonic power transfer has been widely investigated [8] and is beginning to appear in clinical applications [9]. Capacitive power transfer, which can also be named Capacitive
Transcutaneous Energy Transfer (C-TET) for biomedical applications [10],[11], uses a pair of external surface electrodes to generate an electric field, with corresponding pick-up electrodes just below the skin surface to receive power and drive a load as shown in Fig. 1.a. Whilst this method can deliver useful amounts of power to implants near the body surface, the voltage potential applied to deliver power leads to large surface currents if the pick-up is implanted deeply.

Galvanic power transfer technologies have recently been reported which rely on volume conduction to deliver power through the body, with coupling to the skin and implant electrodes via electrodes [12]-[14]. For example, BecerraFajardo et al. used high-frequency burst currents, followed by rectification to extract the envelope and deliver microstimulation of nerves. This technology could be suitable for deep implantation and miniaturisation [15].

An alternative approach is to capacitively couple volume conduction power transfer by applying a dielectric coating to the external and/or implanted electrodes. This replaces the metal-electrode double-layer capacitance with capacitance formed about an insulator. Capacitive electrodes have been proposed as a way to reduce skin irritation in recording applications [16] which may be useful in volume conduction power transfer. At the implant, the application of a dielectric to electrodes is a common technique used to achieve impedance stability, biocompatibility, and corrosion resistance [17]. Furthermore, capacitive coupling has the potential to electrically isolate the power electrodes from other circuits or metallic parts of the device. For instance, metal surfaces (such as a power electrode), have been shown to cause artefacts on neural responses recorded from nearby electrodes [18]. Additionally, coated power electrodes can be used to prevent stimulation currents coupling to the power receiving circuitry due to their high impedance at low frequency. This could prevent the loading of the stimulator, enable the use of current steering approaches [19], or enable simultaneous optimisation of stimulation/recording electrodes and volume conduction power transfer. Therefore, capacitive coupling could aid the development of miniaturised devices incorporating conductive power transfer where many functions must coexist in a small space. 


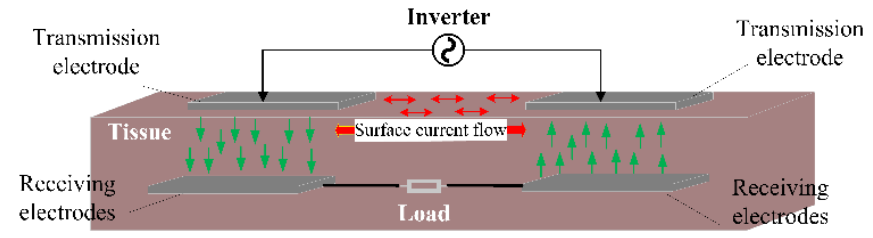

a) C-TET system

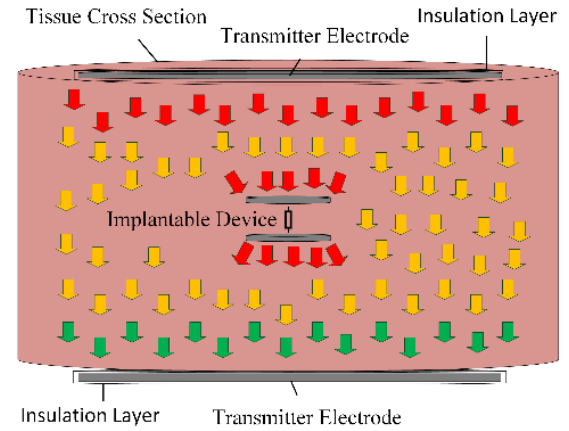

b) Capacitively-coupled conductive TET (CCCTET) system targeting DIBDs

Fig. 1 Conventional C-TET available in the literature vs proposed CCCTET of this work. Arrows illustrate current flow. The electrodes are placed across the tissue with CCCTET to ensure that current is transferred deep in the tissue.

The objective of this work is to investigate the potential of applying a capacitively coupled interface to volume conduction power transfer to supply power to DIBDs. The circuit is configured to apply current uniformly across the tissue bulk, uniformly, as shown in Fig. 1.b. Whilst low coupling causes challenges to power transfer to DIBDs, it allows simplification of the power transfer model, much like IPT [20]. Based on the low coupling simplification and the prior work showing that tissue behaves mainly as a conductor, we then propose a new model of C-TET, namely Capacitively Coupled Conductive Transcutaneous Energy Transfer (CCCTET). This model is used to design example systems which deliver useful amounts of power deep into the body, showing that CCCTET can be used to power deeply implanted devices. In summary, the contributions of this paper are:

1) Developing a circuit model of capacitively-coupled conductive TET which reveals the power transfer mechanism and enables fast determination of output power based on simple measurements.

2) Evaluating the safety of CCCTET through modelling of SAR which shows that the safety is independent of implantation depth.

The paper is structured as follows: in Section II, the system architecture is proposed, and DIBDs' deployment technique is shown. We then analytically model the system at circuit level using simplifications allowed by low coupling (Section III). Section III results in a two-port network. The two-port network allows the determination of compensation network parameters and shows under what conditions maximum power is achieved. This section also introduces the resistive coupling parameter, which has a similar role as the mutual inductance in IPT. The methodology used to validate the analytical models are presented in Section IV. Section V presents the numerical and practical results and compares them with the analytical solution.
Relevant safety standards are discussed, and the safe operating region of CCCTET is introduced in SectionVI. Furthermore, a complete power transfer system is designed and built, and practical issues are discussed in SectionVII and VIII. Finally, the conclusions are presented in Section IX.

\section{SYSTEM DESIGN}

The CCCTET system is depicted in Fig. 2. It consists of a pair of transmitter electrodes (TXE) placed across the body near the implant location (in this example on the back and front of the chest to power a device deep in the patient's torso), an implant, and a control unit (which includes batteries and an inverter). The TXEs can be fabricated metallic electrodes that are insulated to avoid direct skin contact. The receiving electrodes can be placed on or in the immediate vicinity of the implant.

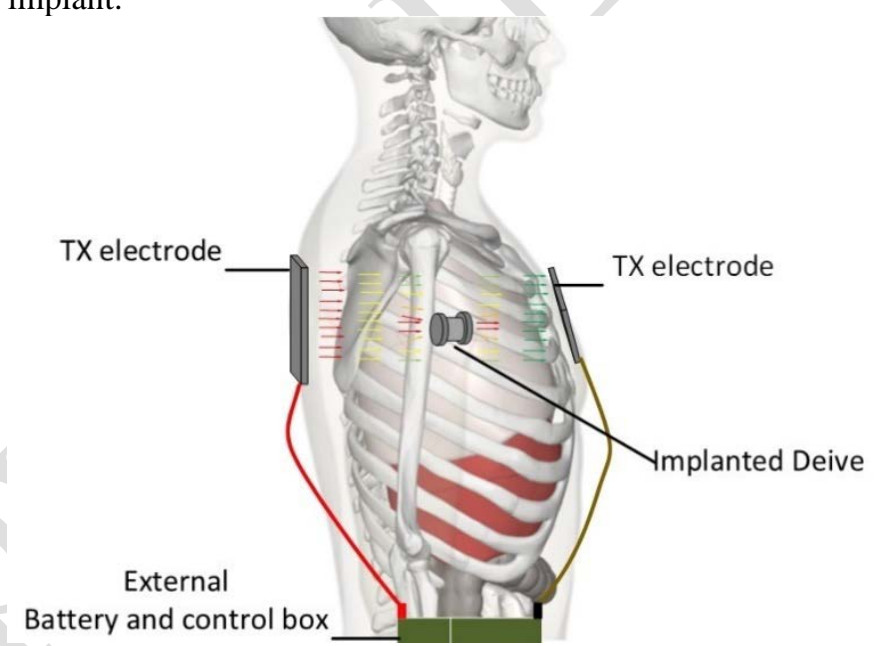

Fig. 2 Practical system representation of the proposed CCCTET for DIBDs. Original image from

https://commons.wikimedia.org/wiki/File:Liver_01_right_side_view.png.

The power transfer system is established via a time-varying electric field (E-field) applied on the TXEs. The E-field generates a current flowing to the implanted electrodes (RXEs) via the partially conductive tissue. The received power is then conditioned and used to drive the implanted biomedical devices.

\section{ANALYTICAL CIRCUIT MODELLING}

\section{A. A simplified circuit model based on conduction}

Fig. 3 taken from [11] illustrates a C-TET circuit model. The schematic includes external matching components $\left(\mathrm{L}_{1}\right.$ and $\left.\mathrm{L}_{2}\right)$, capacitive coupling elements ( $\mathrm{C}_{\text {Co\#-s}}$ ), tissue-equivalent model $\left(\mathrm{R}_{\text {eq\#}}, \mathrm{C}_{\mathrm{TH}-\mathrm{S}}, \mathrm{R}_{\mathrm{TH}-\mathrm{X}}, \mathrm{R}_{\mathrm{TH-}-\mathrm{L}}\right)$ and a resistive load $\left(\mathrm{R}_{\mathrm{L}}\right)$. The tissueequivalent circuit model seen by the electrodes is the starting point for the analysis presented below and is depicted in Fig. 4. In this section of the paper, we will modify the model from Erfani et al.'s model to include capacitive coupling elements, then simplify it based on the resistive properties of tissue to create a two-port model of CCCTET which can be entirely determined by experimental measurements. In order to understand the impact of capacitive coupling reactance on power transfer, an admittance model is developed, which includes tuning inductors allowing the conditions for tuning to 


\section{IEEE POWER ELECTRONICS REGULAR PAPER/LETTER/CORRESPONDENCE}

be determined. Finally, the models are simplified to Thevenin and Norton equivalent circuits for cases of low coupling, as is typical of deeply implanted medical devices.
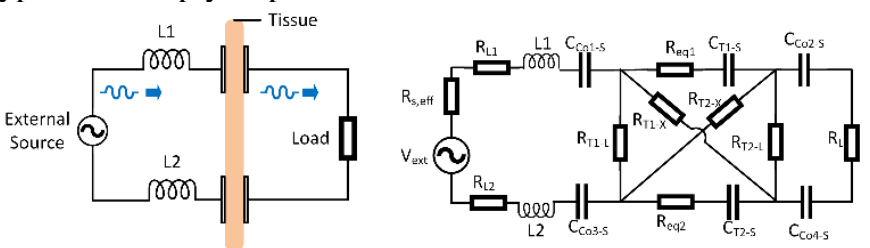

Fig. 3 Circuit model presented by R. Erfani et.al. The left side shows the physical layout with a transmitter, electrode and receiver. The right side is an equivalent circuit model of the left side where the centre cross-over network represents the electrode-tissue interface.

The network in Fig. 4 can be simplified from Fig 3 based on the assumption that tissue is predominantly conductive (phase angle near zero) in the low $\mathrm{MHz}$ range, allowing the reactive components, $\mathrm{C}_{\mathrm{T} 1-\mathrm{s}}$ and $\mathrm{C}_{\mathrm{T} 2-\mathrm{s}}$, be eliminated. The presented circuit model in Fig. 4 can be analysed as three main components, the transmitter, the medium (tissue) and the receiver. The transmitter side consists of TXEs which form $\mathrm{C}_{\mathrm{p}-11-2}$ and $\mathrm{R}_{12}$, the medium includes $\mathrm{R}_{13,23,14,24}$ and finally the RXEs of the receiver side form $\mathrm{C}_{s-11-2}$ and $\mathrm{R}_{34}$. The capacitance formed between the transmitting (TXE) and receiving electrodes (RXE) and the tissue is represented as $\mathrm{C}_{\mathrm{p} \text {-t\# }}$ and $\mathrm{C}_{\mathrm{s} \text {-t\# }}$ respectively. These capacitances are a function of the coating thickness, with the capacitance being formed between the electrode and the tissue across the dielectric material.

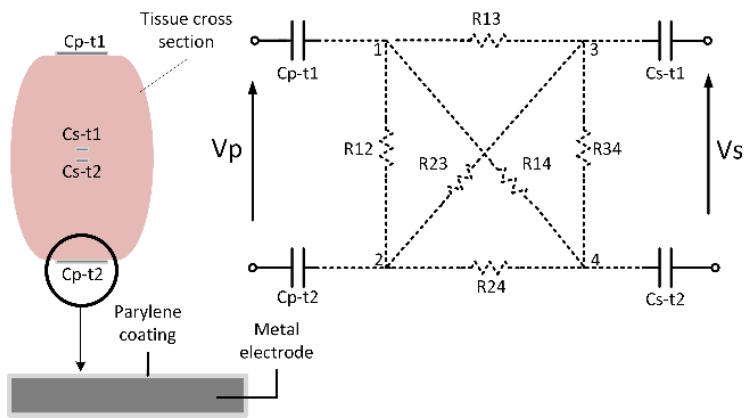

Fig. 4 A general equivalent model schematic of CCCTET consisting of transmitter, receiver and tissue medium with the exclusion of any additional external circuitry.

B. Separation into TX, RX and coupling resistance

By further simplifying the equivalent model illustrated in Fig. 4, the transmitter (TX) and receivers (RX) sides can each be separately analysed, measured, and linked via a coupling resistance.

To find the coupling term to link the transmitter and receiver sides, $\mathrm{R}_{13,23,14,24}$ are grouped into one component, namely $\mathrm{R}_{c}$; the coupling resistance (illustrated in Fig. 5). This term describes how much of the supplied power will be received on the implant side. $R_{c}$ is dependent on the medium's electrical properties, dimensions of the TXEs and RXEs, and separation between the TXEs and RXEs.

The self-capacitors can also be lumped into $\mathrm{C}_{\mathrm{p}-\mathrm{t}}$ and $\mathrm{C}_{\mathrm{s}-\mathrm{t}}$, the values of which can be analytically calculated by equation (1). $\mathrm{C}=\frac{\mathrm{A} \cdot \varepsilon_{0} \cdot \varepsilon_{\mathbf{r}}}{\mathrm{d}}$
Where $\mathrm{A}$ is the surface area of electrodes, $\varepsilon_{0}$ and $\varepsilon_{\mathrm{r}}$ are the permittivity of free space and relative permittivity respectively, and $d$ is the thickness of the insulation layer. The final circuit model is depicted in Fig. 6.
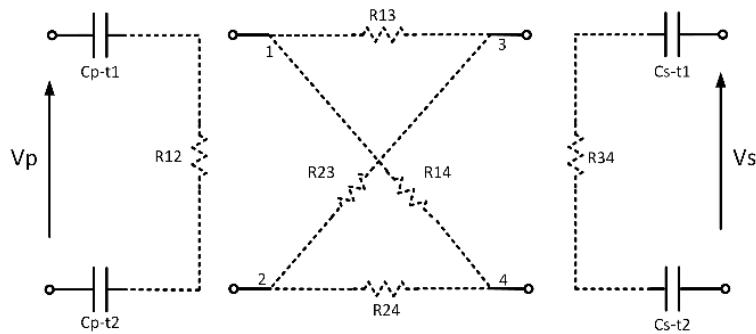

Fig. 5 lumping $\mathrm{R}_{13,23,14,24}$ into one component. The resulting component is an equivalent resistor that links the TX and RX side (coupling resistance). The coupling resistance can easily be calculated analytically as in equation (2).

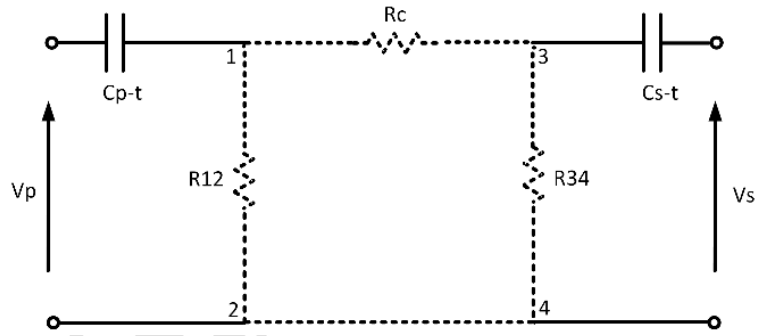

Fig. 6 Resistive equivalent circuit that includes a resistive coupling component

The result is consistent with what has been reported for volume conduction in [21]; however, the proposed model is a Pi equivalent network rather than a $T$ network. This indicates that the capacitive coupling does not change the fundamental power transfer mechanism of volume conduction.

C. Determining the circuit model parameters

$\mathrm{R}_{12}$ can be directly measured using an impedance analyser attached to the TXEs when the RXEs are open-circuited, as shown in Fig. 7 . $\mathrm{R}_{34}$ can be measured similarly by attaching the impedance analyser to the RXEs with the TXEs open-circuited. This measurement method is only applicable to loosely coupled systems such as DIBDs.

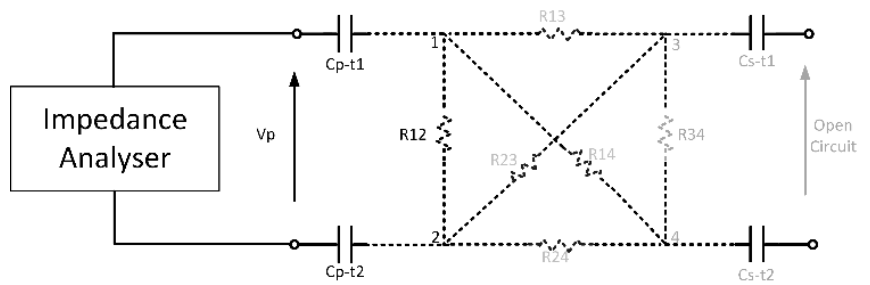

a) Measuring the input impedance with the RXEs open circuited.

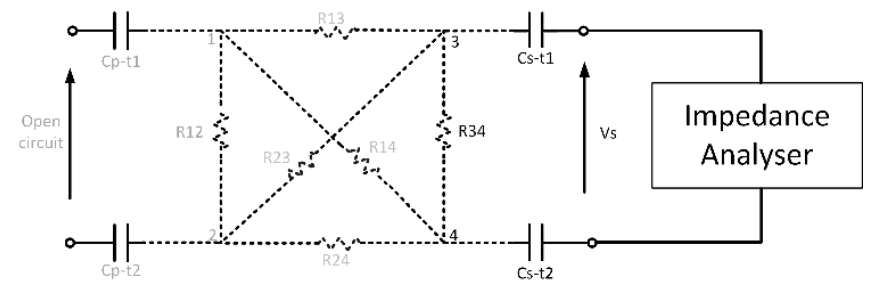

b) Measuring the output impedance, with the TXEs open circuited.

Fig. 7 Impedance analysis steps, first measuring TXEs and then RXEs. $V_{p}$ and $V_{\mathrm{s}}$ represent the connection points to the impedance analyser. 
In practice, measurement of $\mathrm{R}_{\mathrm{c}}$ using an impedance analyser is challenging due to the presence of $R_{12}$ and $R_{34}$ which dominate the impedance measurements. It was found to be more reliable to measure the open circuit output voltage in response to an applied input voltage on the TXEs. Fig 8.a shows the equivalent circuit modified to account for the parasitic inductance and resistance of the electrode connections which must be accounted for when relating $R_{c}$ to the voltage measurements. These are in addition to the coupling capacitance shown in Fig 5, and as such $\mathrm{C}_{\mathrm{p}-\mathrm{t}}$ and $\mathrm{C}_{\mathrm{s}-\mathrm{t}}$, are generalised to impedance $(\mathrm{Z})$ values. Nodal analysis can be used to calculate $R_{c}$ based on the equivalent circuit shown in Fig. 8.a. Determining $R_{c}$ using equation (2) requires a physical measurement of $\mathrm{V}_{\mathrm{s}}$ when there is no load, $\left(\mathrm{V}_{\mathrm{s}}(\mathrm{OC})\right)$. This can be done either experimentally as described above or via numerical simulation.

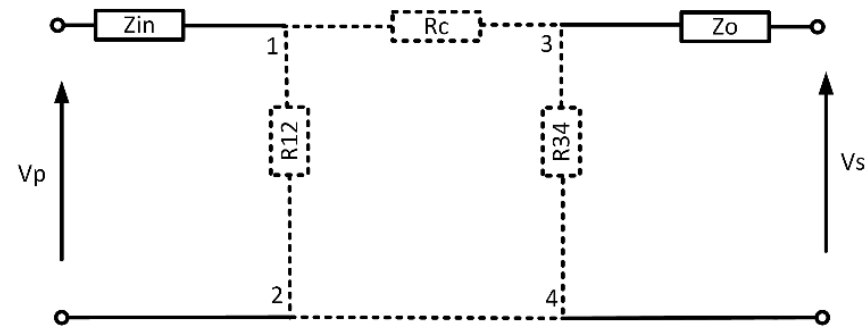

a) Simplified impedance equivalent circuit of CCCTET.

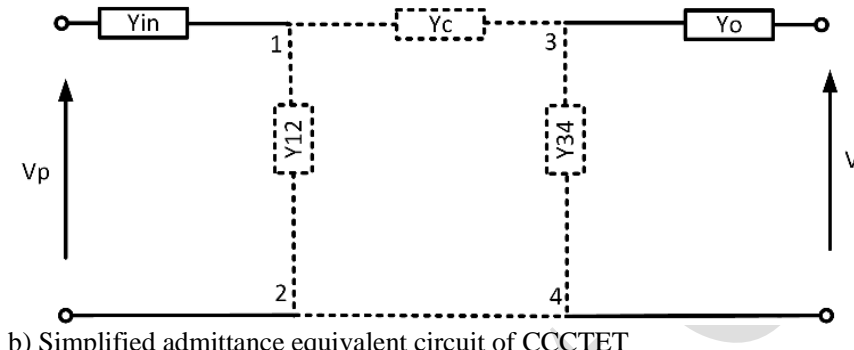

Fig. 8 Equivalent circuits of CCCTET simplifies the design process. Both admittance and impedance models can be used interchangeably.

$\mathbf{R}_{\mathbf{c}}=\operatorname{Re}\left[\frac{\left.\mathbf{R}_{34} * \frac{\mathbf{p}_{\mathbf{p}^{*}} * \mathbf{R}_{12}}{\mathrm{Z}_{\text {in }}+\mathbf{R}_{12}}-\mathrm{V}_{\mathbf{s}(\mathbf{o c})}\right)}{\mathbf{V}_{\mathrm{s}(\mathbf{o c})}}\right]$

The circuit schematic can also be presented by its admittance equivalent (shown in Fig. 8.b) which supports current source analysis, and is used in section II.D. Again, using nodal equations, Yc can equivalently be obtained to determine the coupling admittance.

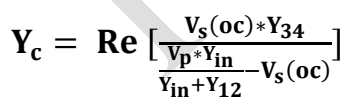

Once $R_{c}$ or $Y_{c}$ are calculated, all the circuit elements are known, enabling all remaining parameters to be found.

D. Admittance model of CCCTET
In this sub-section, we develop an admittance model of the system which is used in section III.E to determine how compensation impacts power transfer, and establish a simple approximation for use in loosely coupled systems. Fig. 9 presents a current fed two-port network. In this network, the transmitting and receiving voltage-controlled current sources are defined as Ips $=\mathrm{V}_{\mathrm{s}} * \mathrm{y}_{21}$ and Isp $=\mathrm{V}_{\mathrm{p}} * \mathrm{y}_{12}$ respectively. $\mathrm{y}_{11}$ and $\mathrm{y}_{22}$ are the transmitting and receiving admittances while $y_{12}$ and $y_{21}$ are the reflected admittances from receiving side to the transmitting side and vice versa, respectively. $\mathrm{Y}_{\mathrm{L}}$ is the load admittance and $\mathrm{P}$ is the load power.

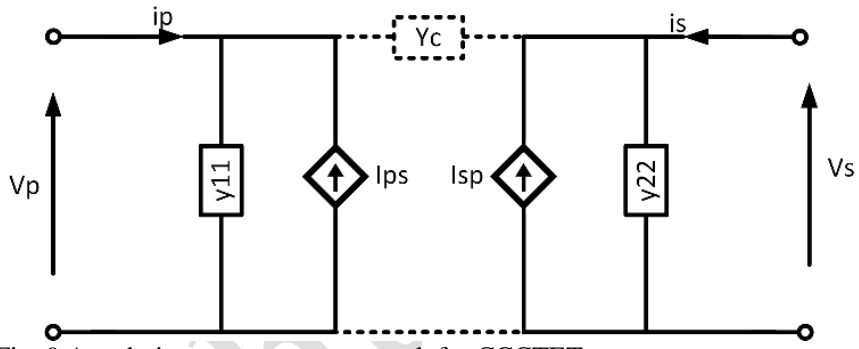

Fig. 9 An admittance two-port network for CCCTET

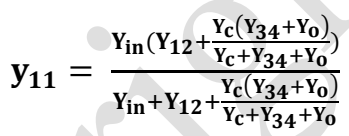

$y_{12}=-\frac{Y_{0} \cdot Y_{\mathbf{c}} \cdot Y_{\text {in }}}{\left(Y_{34}+\frac{Y_{\mathbf{c}}\left(Y_{\text {in }}+Y_{12}\right)}{Y_{\text {in }}+Y_{c}+Y_{12}}+Y_{0}\right)\left(Y_{\text {in }}+Y_{c}+Y_{12}\right)}$

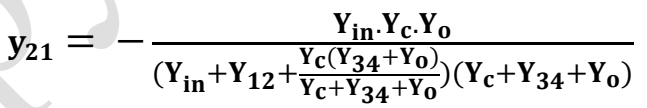

$\mathbf{y}_{22}=\frac{Y_{\mathbf{o}}\left(\mathbf{Y}_{34}+\frac{Y_{\mathbf{c}}\left(Y_{\text {in }}+Y_{12}\right.}{Y_{\text {in }}+Y_{12}+Y_{\mathbf{c}}}\right)}{Y_{\mathbf{o}}+Y_{34}+\frac{\left(Y_{i n}+Y_{12}\right) Y_{\mathbf{c}}}{Y_{i n}+Y_{12}+Y_{\mathbf{c}}}}$

$\mathbf{P}=\frac{\mathbf{I}_{\mathrm{sp}}{ }^{2}}{\mathrm{Y}_{\mathrm{L}}+\mathrm{y}_{22}}$

Each of the parameters shown above is a function of frequency and geometrical size of the system. The geometry of the TXEs and RXEs, as well as their separation distance, will impact the amount of safe power that can be transferred. This will be discussed further in Section VI.

\section{E. Compensation/matching of CCCTET}

In WPT systems, impedance matching is often used to achieve a specific goal. It can be to adjust systems sensitivity (quality factor), generate constant power on the load and or simply compensate for the reactive components [22]. In CCCTET, any of the mentioned targets can be achieved by designing additional circuitry. The easiest and the most obvious case is when the transmitter and receiver capacitive reactance is compensated. If an ideal inductor on either side is assumed, we will have $Y_{i n}=\infty$ and $Y_{o}=\infty$. This condition will dramatically simplify the admittance parameters equations as follows: 


$$
\begin{aligned}
& \mathbf{y}_{11}=Y_{12}+Y_{c} \\
& \mathbf{y}_{12}=-Y_{c} \\
& \mathbf{y}_{21}=-Y_{c} \\
& \mathbf{y}_{22}=Y_{34}+Y_{c}
\end{aligned}
$$

Not only do the parametric equations look a lot simpler but they also they convey a clear message that the interaction between the transmitting side and the receiving side relies heavily on the coupling admittance $Y_{c}$. Therefore, to deliver more power, the coupling must improve. To comply with the loose coupling condition, however, $\mathrm{Y}_{\mathrm{c}}$ shall remain less than $\mathrm{Y}_{12}$. We will revisit this in the next sub-section.

To understand how the TX and RX inductors affect the maximum power transfer, collectively, the following surface plot in Fig. 10 for a system with dimensions similar to Fig. 12 with the respective RXEs and TXEs separation of $15 \mathrm{~mm}$ and $70 \mathrm{~mm}$ was generated. The figure shows the maximum power transferred to a load vs the variation of the tuning inductors in $20 \%$ of its original optimum value (i.e. the inductance required to make resonance). The load was varied to always track maximum power transfer point (MPTP), in accordance with equation (15). The figure highlights the importance of each inductor; illustrating that the system is sensitive to variations of the receiver's tuning circuit. This suggests that the quality factor of the receiver is larger than the transmitter since the capacitance is reduced as a result of the smaller size. The plot also shows a promising $600 \mu \mathrm{W}$ by only applying $2.5 \mathrm{~V}$ peak sinewave at $6.78 \mathrm{MHz}$ on the TXEs.

Maximum Power sensitivity to variation in compesating inductors

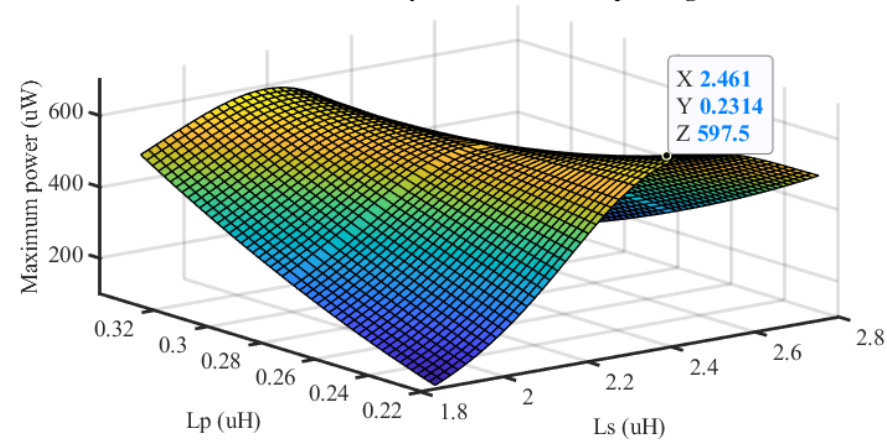

Fig. 10 maximum power transfer as a function of variation in inductance. The values are designed for $6.78 \mathrm{MHz}$ operating frequency, $10 \mathrm{~mm}$ radius RXEs separated by $15 \mathrm{~mm}$ and $50 \mathrm{~mm}$ radius TXEs separated by $70 \mathrm{~mm}$.

\section{F. A loosely coupled system}

In the particular case of loose coupling, the interaction between TX and RX circuits will weaken, meaning the power drawn by the implant is insignificant. Under these conditions, the effect of the RX on TX will be negligible, which will allow each side (RX and TX) to be designed independently of the other. Referring to the two-port network in Fig. 9, the TX and $\mathrm{RX}$ sides become independent, as $\mathrm{Y}_{\mathrm{C}}$ tends to zero (no coupling). For example, if $\mathrm{y}_{11}>>\mathrm{Y}_{\mathrm{c}}\left(\mathrm{Z}_{\mathrm{c}}>>\mathrm{z}_{11}\right)$, when shorting and opening the RX terminals, the impedance change seen at the TXEs will be negligible. In other words, when $\frac{Y_{12}}{\mathrm{Yc}} \gg 1$ (where $\mathrm{Y}_{12}$ is taken from Fig. 8.b) the CCCTET system is considered to be loosely coupled. Therefore, in the case of DIBDs loose coupling conditions are met due to the large separation and small coupling to the implant.

For a loosely coupled system, it is appropriate to replace the coupling admittance with a current-controlled current source, as shown in Fig. 11a. This can be converted to a Thevenin equivalent, as shown in Fig. $11 \mathrm{~b}$.

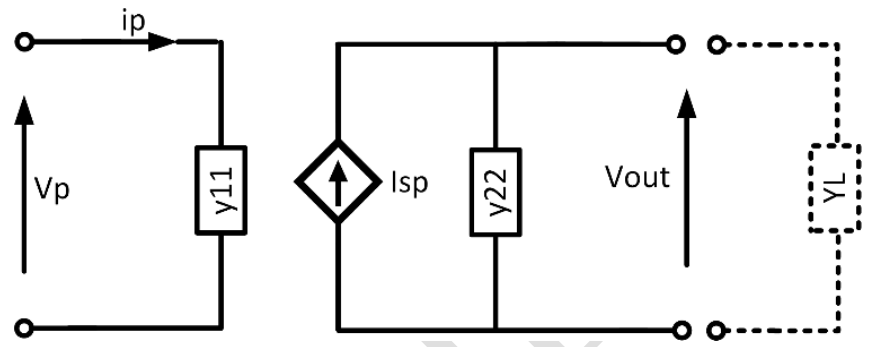

a) Norton network of CCCTET

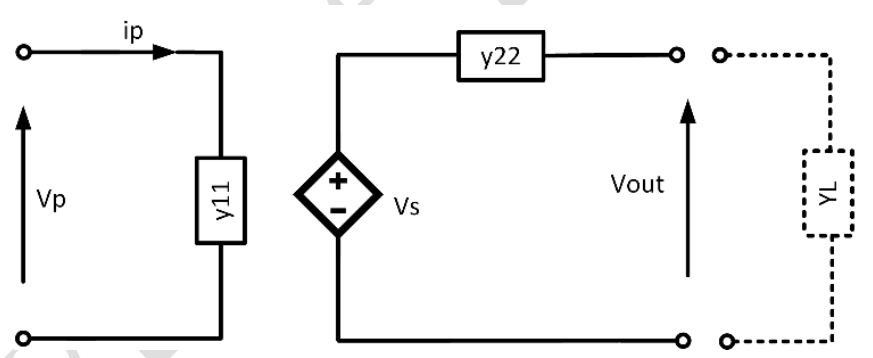

b) Thevenin network of CCCTET

Fig. 11 The loosely coupled two-port network representation of CCCTET. Whilst it is convenient from an analysis point of view to use admittances when analysing conductive power transfer, they can be exchanged for impedances.

In Fig. 11:

Isp $=\mathbf{k}$. ip

Or in the Thevenin equivalent:

$\mathbf{V s}=\mathbf{k V p}$

G. Achieving maximum power transfer in a loosely coupled CCCTET

MPTP for a loosely coupled system is satisfied when the load impedance matches the $R X$ output impedance $\left(R_{L}=R_{22}\right)$. This can be achieved by compensating for any reactance and matching the load resistance to the remaining real component of the impedance. Therefore, the MPTP conditions for the Thevenin and Norton models respectively are:

$P_{\max }=\frac{V_{s(o c)}{ }^{2}}{4 . R_{L}}=\frac{\left(k \cdot V_{p}\right)^{2}}{4 . R_{L}}$

$\mathrm{P}_{\max }=\left[\frac{\left|\mathrm{I}_{\mathrm{sp}}\right| \cdot \mathrm{Y}_{\mathrm{L}}}{\mathrm{y}_{22}+\mathrm{Y}_{\mathrm{L}}}\right]^{2} * \frac{1}{\mathrm{Y}_{\mathrm{L}}}=\frac{\left|\mathrm{I}_{\mathrm{sp}}\right|^{2}}{4 \mathrm{Y}_{\mathrm{L}}}$

The equations in sub-section $F$ and $G$ above show that $a$ loosely coupled CCCTET can be characterised by measuring the TX impedance, the RX impedance and measuring the opencircuit voltage. The TX and RX can then be tuned individually to compensate for the reactance at the desired operating 


\section{IEEE POWER ELECTRONICS REGULAR PAPER/LETTER/CORRESPONDENCE}

frequency. The load resistance can then be set to match the real component of the RX impedance to achieve maximum power transfer. The result is equivalent to the design approach that is commonly applied to inductive power transfer systems under loose coupling conditions [23]

\section{Simulation and experimental setup}

A COMSOL Multiphysics simulation study and practical experiments were conducted to validate the analytical circuit model presented in the previous section. In this section, the simulation and experimental setup are discussed.

A. COMSOL Multiphysics: Finite element and circuit analysis COMSOL simulations were performed to determine safety by calculating the power deposited in the tissue as SAR. A COMSOL model was also developed for comparison with the analytical and experimental power measurements to give further validation of the models' accuracy.

Fig. 13 illustrates the setup used to model CCCTET in the COMSOL environment using the Conductive Currents formulation in the AC/DC module. TXEs and RXEs were placed in the tissue volume matching the tissue saline phantom in the next sub-section. The materials were selected to be Titanium, coated with Parylene for TXEs and RXEs and 0.09 $\mathrm{M}$ saline as the medium, using data from our previous work [24]. The simulation dimensions were identical to those used in the experimental verification, and the Parylene thickness was set to $30 \mu \mathrm{m}$ as measured by ultrasound on the experimental coated electrodes. The model was set such that all the dimensions shown could be controlled. The electrical properties of the medium were frequency-dependent based on the Debye model [25].

Circuit analysis toolbox was utilised to combine field and circuit analysis. To do so, TXEs were connected to an AC sinewave source, the amplitude of which was set to match the practical experiments. A load resistor was connected to the RXEs. Additional parasitic components, such as lead inductance, resistance, and capacitance were included in the model as circuit elements with the values determined by measurements using an impedance analyser. Tuning inductors and capacitors were added to compensate for the electrodes' coupling capacitance due to the Parylene coating.

To calculate $Y_{12}$ and $Y_{34}$ values, a voltage source was connected on the TX and RX electrodes, respectively. By measuring the current through and the voltage across the source, these parameters were determined. The coupling admittance $Y_{c}$ was then found by using $Y_{i n}, Y_{12}$ and $Y_{34}$ data in accordance with equation (3).

The maximum power transfer point was verified by 1 ) varying the load resistance and recording the received power across the load; and 2) by shorting and opening the RXEs to find the short circuit current and open-circuit voltage respectively. The latter results in the admittance seen across RXEs ( $\left.y_{22}\right)$ whereby the maximum power point can be found by equating $\mathrm{Y}_{\mathrm{L}}$ to the real part of $\mathrm{y}_{22}$.

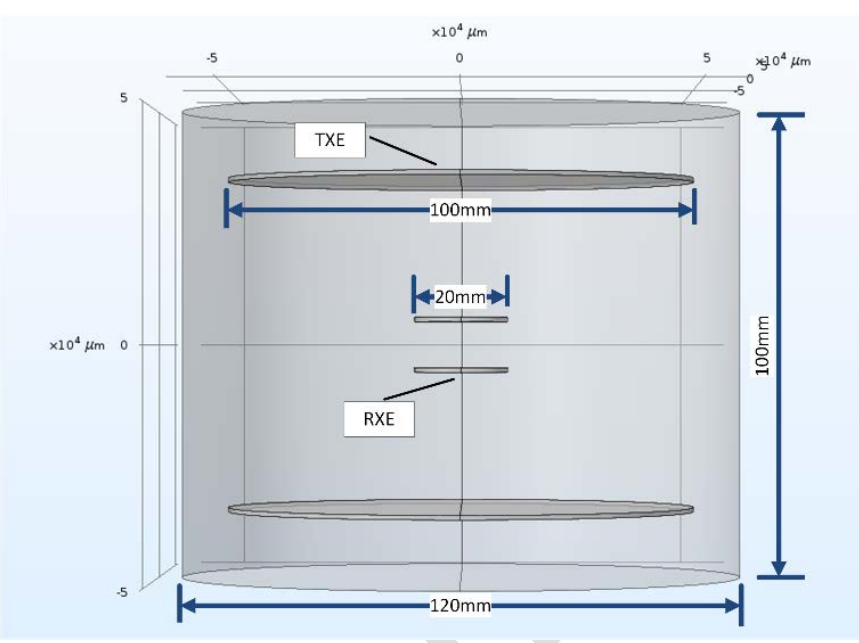

Fig. 12 The geometry setup in COMSOL Multiphysics environment. The dimensions match the ones in practice. Any sweep in the geometry is done on this model.

B. Experimental setup

The experimental setup shown in Fig. 13 was created to match the one in COMSOL. The test rig consisted of:

1- A circular acrylic tube used as a container to hold the tissue phantom fluid

2- A large pair of circular Parylene coated titanium electrodes used to model the TX plates

3- A small pair of Titanium circular Parylene coated electrodes used to model the RX plates

4- Acrylic electrode holders, designed to have minimal impact on the measurements which was achieved by making their cross-section as small as possible

5- $0.09 \mathrm{M}$ saline as the tissue phantom

6- CS1070 Power Amplifier from Cleverscope (https://cleverscope.com/)

7- CS448 Oscilloscope from Cleverscope

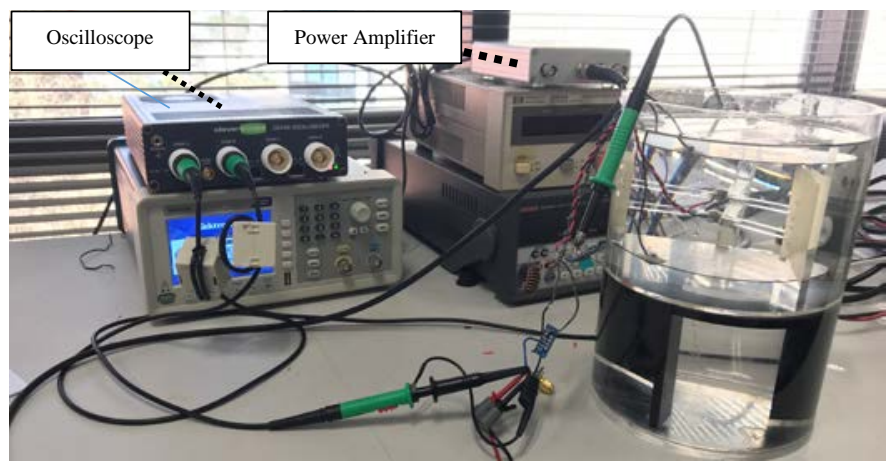

Fig. 13 The experimental setup designed to validate the analytical equations. Cleverscope oscilloscope and power amplifier are shown in the figure.

A saline phantom was used instead of post-mortem tissue. Because firstly, it allows for geometry variation, secondly it can be replicated, and its properties are well known, allowing easy integration into the COMSOL model and finally post-mortem tissue conductivity varies markedly from its living counterpart 
[26]. To prepare the saline, UV treated deionised water (Millipore Direct-Q) was mixed with laboratory-grade $\mathrm{NaCl}$ (Scharlau SO0225025P) to a concentration of $90 \mathrm{mM}$ to mimic the conductivity of muscle [27]. The titanium electrodes for the experiment were laser cut from a titanium grade II sheet (McMASTER-CARR). After surface preparation, leads were attached using nuts and bolts (solder does not stick to Titanium). The electrodes were then coated with Parylene (PDS2010). Once coated, the thickness of the coating layer was measured to be between $26 \mu \mathrm{m}$ and $30 \mu \mathrm{m}$. Parylene was chosen due to its track record as a bio-stable biocompatible polymer which is often used in implantable applications [28]. Additionally, Parylene is vapour-deposited leading to uniform coating thickness, dielectric properties and capacitance values. The thickness is a compromise between achieving a mechanically robust coating and the slow speed of the vapour deposition process.

An acrylic rig was designed to hold the TXEs and RXEs in a precise position while placed in a cylinder filled with saline.

With the electrodes and test rig setup, Keysight E4990A was used to investigate the impedance response of the setup in order to find the parameters, as described in Section III.B. To ensure precision, the device was calibrated according to the Keysight instructions. Depending on the geometry of the experiment, the values of the tuning inductors and capacitors were derived. To generate the voltage on the TXEs, a linear power amplifier, CS1070 was driven by a sinusoidal signal source from the oscilloscope.

The RXEs are floating with respect to ground, and of relatively high impedance, an isolated oscilloscope with low capacitance to ground was used to make measurements (Cleverscope CS448). Ferrite chokes were used to reduce common-mode noise on the signals. Long ground loops were avoided, and the probe lead was carefully routed away from earthed test equipment to minimise parasitic coupling. During the measurement, the probe was attached to the load in order to eliminate the capacitance to the ground that holding the probe would cause. The load resistor was a Vishay Precision metal foil potentiometer, Accutrim ${ }^{\mathrm{TM}} 1240$ (Digikey part numbers: 804-1004-ND). This type of trimmer allows for stable load values and it offers $80 \mathrm{nH}$ of inductance, making it suitable for AC power measurement.

For each set of geometries, the following procedure was performed:

1- The separation distance of RXEs was set as required.

2- The self-resonance frequency of the TXEs was tuned to $6.78 \pm 0.1 \mathrm{MHz}$ using tuning inductors and capacitors.

3- The admittance parameters were measured and calculated at $6.78 \mathrm{MHz}$ using the impedance analyser.

4- The setup was powered using the linear power amplifier. The amplifier's output amplitude was measured using the oscilloscope.

5- $\quad V_{s}(0 c)$ was measured across the RX plates, and $Y_{c}$ was calculated based on the impedance measurements.
6- The load was connected and power was measured as a function of load resistance.

V. Analytical circuit Model Verification Results

The verification results for a test setup of $50 \mathrm{~mm}$ radius TXEs, $10 \mathrm{~mm}$ radius RXEs, $70 \mathrm{~mm}$ TXE separation and $15 \mathrm{~mm}$ RXEs separation placed in $0.09 \mathrm{M}$ saline is presented here. TABLE 1 includes the parameters as measured using the impedance analyser and calculated using the analytical equations (8) discussed previously. The parameters' values suggest that $\frac{\mathrm{Y}_{12}}{\mathrm{Y}_{\mathrm{c}}} \gg 1$, meaning the system is loosely coupled (refer to section III.D for more details). Loose coupling is achieved as expected due to the geometry of the system, i.e. deeply inserted RXEs.

The waveforms in Fig. 14, taken from benchtop measurements, illustrate the voltage measurements at the input to the transmitting terminals and output of the receiving terminals. In this figure, channel A (in red) refers to the load voltage while channel B (in blue) illustrates the input voltage at the TXEs. The sinusoidal waveforms of channel A and B contain a low level of harmonics. Low harmonics are achieved by using a linear amplifier to amplify the arbitrary waveform created by the signal generator. It is necessary to have low harmonics because the presented equations are all based on the fundamental frequency, and therefore, harmonics will result in a mismatch of the results. TABLE 2 compares the data taken from each model to the practical measurement. A reported error of $4 \%-10 \%$ is recorded. Using practical measurement as the reference allows the models to be verified in real world conditions. The plot of Fig. 16 illustrates the analytical (in red), numerical (in magenta) and experimental (in blue) results of power measurement with respect to the variation of load resistance. The solid red line is the loosely coupled model calculated from equation (14). The analytical model (red boxes) was evaluated from equation (8). Both were calculated by substituting experimental impedance and coupling measurements into equations (8). The numerical data was taken directly from COMSOL Multiphysics. The plotted data points show a reasonable match regardless of the difficulties and uncertainties in low power measurements.

TABLE 1

Measured Circuit Parameters for 10mm RXE and 50mm TXE at 27.5mm

\begin{tabular}{ccc}
\multicolumn{3}{c}{ Separation } \\
\hline \hline Parameter & Experimental & COMSOL \\
\hline \hline Y12 (S) & 0.11 & 0.13 \\
Y34 (S) & 0.053 & 0.05 \\
Cs,self (pF) & 268 & 270 \\
Yc* (S) & 0.014 & 0.012 \\
RXE leads inductance Ls (nH) & 522 & $522^{* *}$ \\
Probe Capacitance (pf) & 10 & $10^{* *}$ \\
Source output impedance (ohm) & 1 & $1^{* *}$
\end{tabular}

*Calculated based on the input and open-circuit voltage

** Added to COMSOL based on measurement 
TABLE 2

Load voltage error rate with respect to practical measurement. Analytical values are taken from equation (8) by substituting practical measurement, while numerical data is obtained from COMSOL.

$\begin{array}{cccc}\text { Load (ohm) } & \begin{array}{c}\text { Vout } \\ \text { (Measured) }\end{array} & \begin{array}{c}\text { Analytical Error } \\ (\%)\end{array} & \begin{array}{c}\text { Numerical Error } \\ (\%)\end{array} \\ 150 & 0.343 & 4 & 6 \\ 100 & 0.334 & 3 & 5 \\ 80 & 0.304 & 6 & 4 \\ 50 & 0.289 & 4 & 4 \\ 30 & 0.263 & 4 & 7 \\ 10 & 0.237 & 3 & 3\end{array}$

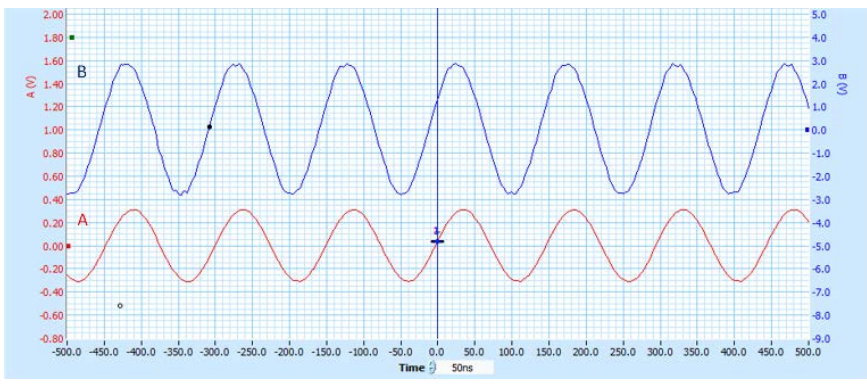

Fig. 14 Input voltage (channel B in blue) and load voltage (channel A in red).

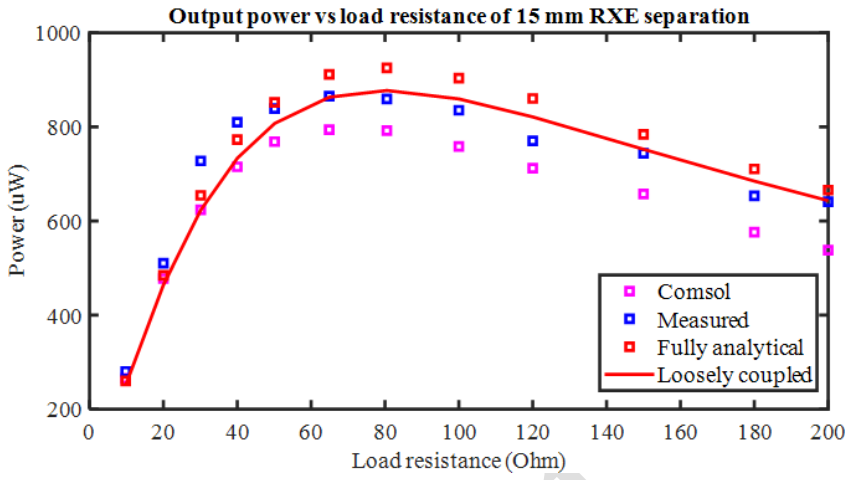

Fig. 15 Comparison of the predicted output power as the load resistance is changed from COMSOL simulation, Full Analytical model equation (8), and loosely coupled model (15) compared to the measured power from a compensated TX circuit with partially compensated RX. Analytical models use impedances and coupling parameters measured on the experimental setup.

\section{SAFETY}

IEEE C95.1 set limits on the magnitude of the electric field (E-field), and Specific Absorption Rate (SAR) allowed as a function of frequency [29]. The primary limitation of CCCTET is tissue heating due to current flow. Specifically, in CCCTET, the standard limits the maximum possible exposure of RMS Efield in the frequency of 1 to $30 \mathrm{MHz}$ to $1842 / \mathrm{f}_{\mathrm{M}}\left(\mathrm{f}_{\mathrm{M}}\right.$ is the operating frequency in $\mathrm{MHz}$ ). The basic restriction of localised (peak spatial average) SAR is limited to $2 \mathrm{~W} / \mathrm{kg}$ averaged over $10 \mathrm{~g}$ of tissue (refer to TABLEs 6 and 8 in the standard). SAR and E-field are interrelated by equation (16).

$\mathbf{S A R}=\frac{\sigma * \mathbf{E}^{2}}{\rho}$

Where $\sigma$ is materials conductivity in $\mathrm{S} / \mathrm{m}, \mathrm{E}$ is the E-field strength in $\mathrm{V} / \mathrm{m}$ and $\rho$ is the density of the material in $\mathrm{kg} / \mathrm{m}^{3}$. In tissue at the frequency of interest $(6.78 \mathrm{MHz})$, SAR limits are reached at an equal or higher rate than E-field limits. This is because the conductivity of tissue reaches almost $1 \mathrm{~S} / \mathrm{m}$ and the density is very close to that of water, $1000 \mathrm{~kg} / \mathrm{m}^{3}$. This simplifies the SAR equation to $\mathrm{SAR}=\mathrm{E}^{2} / 1000(\mathrm{~W} / \mathrm{kg})$. Therefore, the primary focus of this section will be SAR which was evaluated in COMSOL.

\section{A. Methodology}

SAR is calculated in accordance with IEEE C95.1 and COMSOL guideline [30]. Fig. 16 demonstrates the volumetric loss density plot in the COMSOL environment (postsimulation). The figure includes important information that will be described. Briefly, a 10 g cubic volume with equal length is created. The power loss is averaged inside the cube by volumetric averaging. The cube is displaced to capture the volume with maximum power loss. When RXE radius is varied, so is the electrode to tissue capacitance. Therefore, tuning inductance has been altered to ensure full compensation. When altering RXE separation, TXE separation is kept fixed and vice versa. For each of the presented cases, the load that results in MPTP is calculated and the amplitude of the TXE source is adjusted until the SAR limit ( $2 \mathrm{~W} / \mathrm{kg}$ ) is reached. Finally, the power delivered to the load is recorded as the maximum safe power rating.

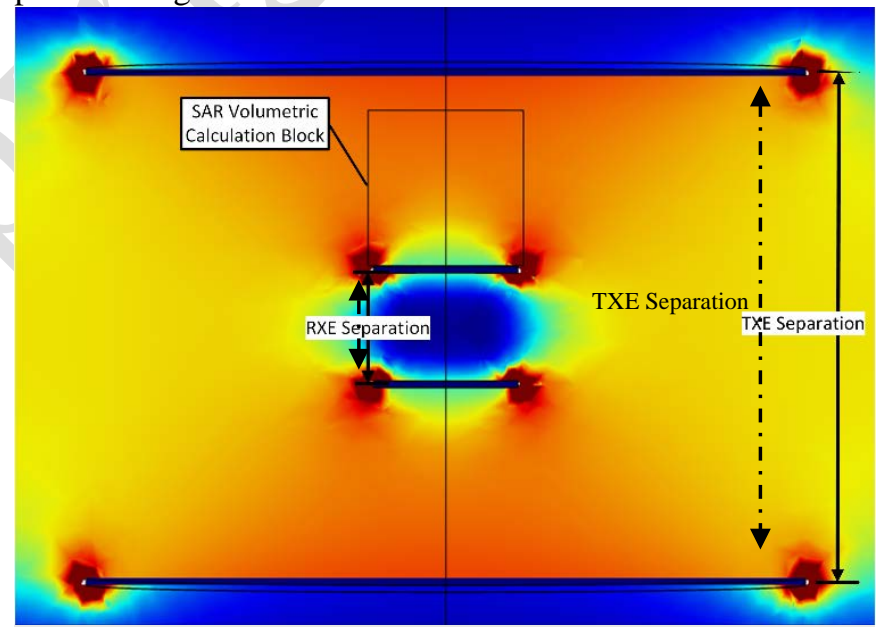

Fig. 16 Volumetric loss density plot of the COMSOL simulation. The SAR volumetric calculation block (10 g averaging) is displaced to capture the maximum SAR. RXE separation and TXE separation, which will be used in the following safety plots are illustrated.

\section{B. Results}

The results illustrated in Fig. 17.a,b,c are obtained in COMSOL for a fully tuned system operating at the maximum power transfer point. The highlighted area under each plot shows the safe operating region. The results in Fig. 17.a show that as the separation distance between RXEs increases, the safe power level also increases. While Fig. 17.b illustrates larger receiving electrodes result in increased power delivered to the load safely. This phenomenon is related to the E-field density and distribution as SAR is directly linked to the magnitude of E-field. Therefore, from the design point of view, one has to redistribute the E-field for small separations (e.g. by using a 


\section{IEEE POWER ELECTRONICS REGULAR PAPER/LETTER/CORRESPONDENCE}

larger radius) in order to maximise the safe power output. In the examined cases, as the distance between RXEs decreases, the coupling admittance drops, resulting in a lower induced voltage on the electrodes with no real drop in SAR. Consequently, the power available is lower for tightly coupled electrodes. Fig. 17.c demonstrates that the safe power level is almost constant as TXEs are moved further apart. In other words, the safe power delivered to the implant is not affected by the implantation depth. In short, the results are indicative of useful amounts of power at a considerable separation distance, considering that most biosensors and pacemakers require less than $1 \mathrm{~mW}$ of power [31].

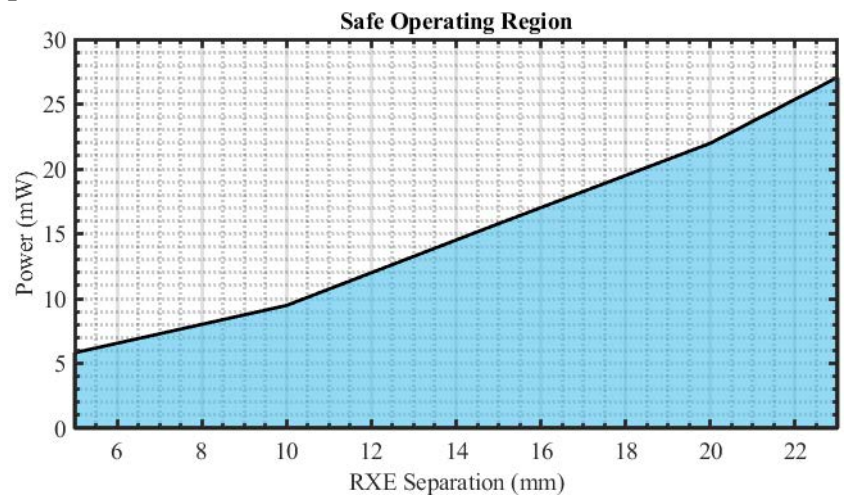

a) Maximum safe power vs RXE separation for $10 \mathrm{~mm}$ radius RXE.

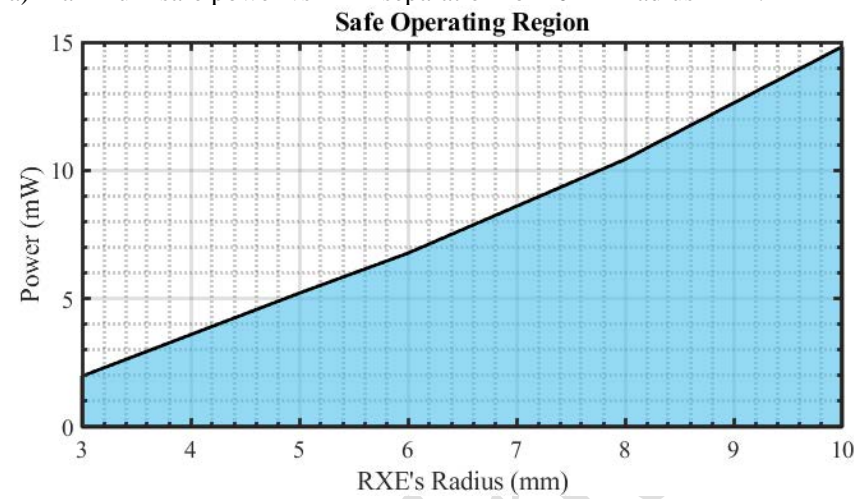

b) Maximum safe power vs RXE radius at $27.5 \mathrm{~mm}$ separation between RXE and TXE.

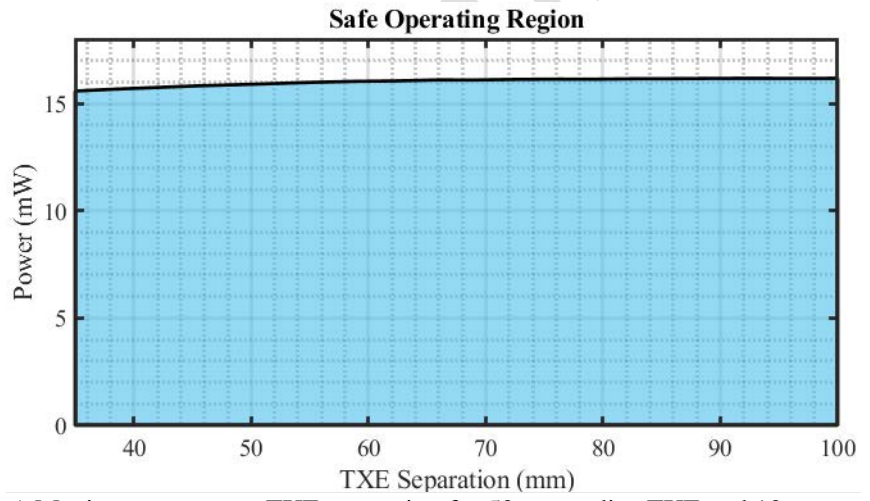

c) Maximum power vs TXE separation for $50 \mathrm{~mm}$ radius TXE and $10 \mathrm{~mm}$ radius RXE. RXE separation is fixed at $15 \mathrm{~mm}$. The safe operating region defined by SAR limits in IEEE C95.1 using $2 \mathrm{~W} / \mathrm{kg}$ limits.

Fig. 17 Safe operating region calculated in COMSOL for fully compensated CCCTET system.
VII. A FULLY COMPENSATED SAFE CCCTET IMPLEMENTATION

In the previous sections, experimental results were used to verify and validate the analytical and numerical models. In this section, a fully compensated CCCTET system working in the safe operating region is presented. The methodology of the experiment follows similar guidelines to Section IV.

The electrode to tissue capacitance of TX is compensated with an addition of a $1.6 \mathrm{nF}$ C0G capacitor and $428 \mathrm{nH}$ inductor. The capacitance of the receiving side $(\mathrm{RX})$ is compensated with a $2.3 \mu \mathrm{H}$ inductance wound around a toroid core (Fair-Rite 5967000601). By utilising the impedance analyser, full compensation condition has been confirmed. Because the CS1070 power amplifier is limited to $1 \mathrm{~A}$ output current, the voltage output has been lowered to avoid clipping.

Fig. 18 shows the plot of the variation of load for $10 \mathrm{~mm}$ $\mathrm{RXE}$ and $50 \mathrm{~mm}$ TXE radii fixed at $75 \mathrm{~mm}$ (TXE-Implant) separation. The voltage input is set to $6.4 \mathrm{~V}_{\mathrm{pk}-\mathrm{pk}}$ sinewave operating at 6.7 MHz. The resulting RMS E-field is $32 \mathrm{~V} / \mathrm{m}$, which is $12 \%$ of the maximum possible exposure outlined in IEEE C95.1. The analytical circuit, loosely coupled model, and numerical data have also been overlaid for validation. The results show $10 \mathrm{~mW}$ of power delivered with an input power of $2.2 \mathrm{~W}$ and an efficiency of $0.45 \%$ (TXE to RXE). The CS1070 linear power amplifier has $4 \mathrm{~W}$ of dissipated power, which has been neglected here given that in practice, a high-efficiency inverter would be used [32]. The majority of the energy to generate a voltage on the RXEs is dissipated in the saline tank, which corresponds to SAR and tissue heating. Despite its low efficiency, the system is compatible with safety standards.

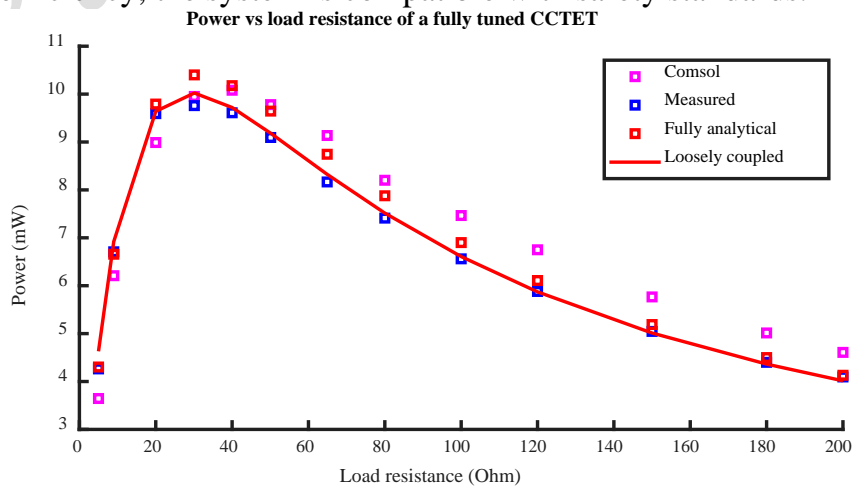

Fig. 18 Power vs load variation for a fully compensated TX and RX at $75 \mathrm{~mm}$ separation. Analytical data is taken from equations (8). The plot shows a lower bandwidth compared to Fig. 16 that illustrates the effect of compensation.

\section{DISCUSSION}

The CCCTET system presented in this paper offers an appreciable amount of safe power to be delivered deep in the tissue. The validated analytical and numerical models permit ease of analysis and construction of a practical system. Here some key aspects of the paper are discussed.

The analytical model is simple, easy to use and insightful due to the Conductive nature of the tissue. The presented model ignores the capacitive component of tissue. It also sums up the coupling into one resistive coupling that can be precisely modelled and measured. The result is a two-port network that 
allows the decomposition of TX and RX.

The loosely coupled model is simple and accurate. The model assumes weak coupling between the TX and RX side. This means that the RX circuit will not affect the TX side. This permits multiple RX with various geometries to be implanted. The model also allows a compensation circuit to be designed and the maximum power load to be calculated easily.

Safety of power transfer (within the SAR limits) is independent of depth, as shown in figure Fig. 17.c. Given the volume conduction basis of the method, we would expect the same to apply to galvanically coupled systems, though this would need to be confirmed through further investigation.

The most recent version of IEEE c95.1 standard published in 2019 suggests that stimulation of cells while highly probable in low frequency is less likely at high frequency and no stimulation restrictions exist above $5 \mathrm{MHz}$. The standard further emphasises that stimulation of cells is more likely when pulsed sources are used compared to continuous waves [33]. The choice of $6.78 \mathrm{MHz}$ operating frequency running in continuous mode allows this implementation of CCCTET to be exempt from the IEEE c95.1 restriction.

The size of the power transmitter and receiver should be considered as they both relate to usability. Inductively powered systems have been proposed for high power and depth. For instance, Puers et al.'s work includes a $150 \mathrm{~mW}$ miniature receiver while the transmitter is wound around the entire upper body [34]. More recently, Ghovanloo et al. and Meng et al. have proposed miniaturised transmitter and receivers $\left(1 \mathrm{~mm}^{3}\right)$ which operate at $3 \mathrm{~cm}$ whilst delivering $200 \mu \mathrm{W}$ [7], [35]. Other authors such as Maharbiz et al. had to include a transcutaneous repeater to target $15 \mathrm{~mm}$ deep brain tissue which adds to the size and complexity of the system [36]. Neither of these IPT devices is compatible with metallic hermetic sealing (e.g. titanium canister) used in almost all implants, which is a significant barrier to encapsulation and reliable use in humans [37], [38]. On the other hand, ultrasonic powered technologies are compatible with metallic implants and can achieve larger distances compared to IPT; however, the power density is low. For instance, Charthad et al. could achieve $100 \mu \mathrm{W}$ over $10 \mathrm{~cm}$ to a device of $40 \mathrm{~mm}^{3}$ volume [8]. Similarly, galvanic power transfer can be used to power metallic devices, and the work of Ivorra et al. proves that $1 \mathrm{~mW}$ can be transferred over $6 \mathrm{~cm}$ [13]. In this work, $10 \mathrm{~mW}$ has been safely transferred over $75 \mathrm{~mm}$ with an effective surface area of $628 \mathrm{~mm}^{2}$ and a volume of under $600 \mathrm{~mm}^{3}$.

Fig. 19 compares the discussed technologies with this and other contemporary works. To create the figure, the power delivered to an implant was divided by the size of the pick-up (for example, the electrodes, in this case, antenna for radiative systems or the pick-up inductor for IPT) of that implant to obtain the power density of each system. Power density is plotted against the implantation depth, which is the distance from the transmitter to the implant. In the case of systems with repeaters, the distance between the repeater and the implant was selected, and the repeater was the closest large power component to the implant. In [34], the study considered wholebody SAR only, and safety limits were violated in some limb positions.

The power we present in theory can be achieved using much thinner electrodes. In this work, $0.9 \mathrm{~mm}$ titanium sheet was used due to the requirements of laser cutting, however much thinner electrodes could, in theory, be used. In order to stimulate the research community to consider CCCTET or related volume conduction techniques, we have added a "Future" power density figure which is based on $0.1 \mathrm{~mm}$ thick electrodes.

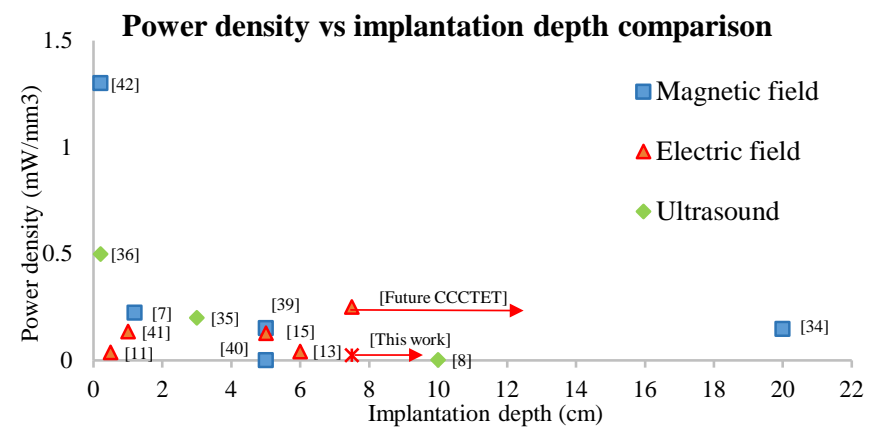

Fig. 19 A comparison of existing TET systems taken from [7], [11], [13], [15], [20], [35], [36], [39]-[42]. The red arrows illustrate that achievable CCCTET power is independent of implantation depth when SAR is the limiting factor. This indicates that the implant could operate at greater depth at the same power whilst remaining safe, as shown in the figure.

Linear power amplifiers contribute to significant losses; therefore, a switching converter will be an ideal choice as linearity is not required in a practical setup. Harmonics filtering can be added to a power converter if required. In certain applications, pulsed power supplies can be an alternative method. With pulsed systems, care must be taken to ensure average safety limits are maintained in the basic restriction band.

The presented model here comes with limitations. 1) the tissue is assumed to be cylindrical for the experiment which may not resemble the real geometry of a person. The cylindrical setup, however, is a suitable way to verify the analysis and ensure repeatability 2) The model uses homogenous tissue and does not account for tissue variation. 3) The analysis shows a strong coupling between TXEs to the tissue which can be achieved by applying a conductive gel in practice.

In summary, the modelled and measured results both in Fig. 15 and Fig. 18 show a reasonably good match. There is a maximum error of $10 \%$ between the modelled and measured results. The reason for the discrepancy is there are a number of variables involved in the equations that contribute to the overall error rate. A designer may wish to match the models and practical experiments via the addition of parasitic components. Authors suggest this practice only after a complete system has been finalised.

Furthermore, the validated models can serve as design guidelines to develop various shapes and geometries of CCCTET without the need to create practical systems. Briefly, the following steps are suggested:

1- Select the desired geometry: The geometry can be 
decided based on the targeted implantable device.

2- Use COMSOL to calculate the circuit parameters

3- Utilise the circuit model of Fig. 11 to design additional circuitry as required.

Following these steps will result in a fully functional circuit model that can be simulated in Spice programs. Tuning circuits, inverters, rectifiers and control systems can be designed accordingly.

Like other WPT technologies, CCCTET has its advantages and disadvantages. For instance, to attain maximum power transfer, CCCTET requires a tuning inductor to cancel the reactive impedance of the electrode capacitance, which consumes volume in the device. However, such an inductor should be relatively small, for instance, inductors with a suitable current rating for the system presented here are available in an 0402 package $(1.0 \times 0.5 \mathrm{~mm})$. This inductance also tunes the pick-up circuit, slowing transient response which may reduce data rate in applications where the power transfer is modulated to transmit information to the implant [43]. However, the tuning can be an advantage if used to boost the received voltage [44] or equivalently as impedance transform to match the pick-up electrodes to a particular load [23].

In this work, a simple parallel plate geometry was used to simplify the numerical modelling and the experimental set up. This led to a rather large prototype which could be reduced by using thinner electrodes. Recent work has shown that long aspect ratios are very effective in volume conduction systems [15], which could be utilised in future capacitively coupled systems to reduce size and increase power density.

CCCTET is particularly useful for miniaturised metallic devices exemplified by vascular neuromodulation implants such as the Stentrode [45]. The Stentrode utilises a stent body to hold electrodes against a vessel wall in the brain. In this application, the nitinol stent body could be capacitively coupled to volume conduction to form an electrode for power transfer whilst reducing the impact of the power transfer system on recording or stimulation functions. This would require electrically separating the stent body into two sections such that a voltage difference could be generated for power transfer.

In the future, the limitations of the current study will have to be overcome in order to develop practical applications of CCCTET. For instance, the numerical models presented here are quasi-static in nature and assume that the skin depth is large in tissue and therefore, insignificant [15]. This may not be the case in highly conductive electrodes and conductors where the skin depth could be significant. Therefore, practical implementations should consider the impact of proximity effects which may be significant in some configurations. Further, future modelling work will need to address the impact of human anatomy, which includes realistic tissue distributions and impedance properties.
IX. CONCLUSION

In this paper, a Capacitively Coupled Conductive Transcutaneous Energy Transfer (CCCTET) method based on conductive tissue to power deeply implanted biomedical devices (DIBDs) is presented. Simplified analytical models are validated by comparison with numerical analysis and tissue phantom measurements. A prototype system delivering more than $10 \mathrm{~mW}$ of power at $0.45 \%$ efficiency to a deeply implanted load has been constructed. The promising safety results show that safe power transfer is independent of depth which is highly desirable for DIBDs. Given the feasibility of CCCTET, future studies will include heterogeneous tissue models and animal studies.

\section{ACKNOWLEDGMENT}

The authors would like to thank the University of Auckland, New Zealand and Chongqing University, China for providing access to the experimental and computational laboratories. We would also like to acknowledge Davella Tang's and Quinn Boesley's efforts in proofreading the article.

\section{REFERENCES}

[1] H. Matsuki, 'Energy transfer system utilizing amorphous wires for implantable medical devices', IEEE Trans. Magn., vol. 31, no. 2, pp. 1276-1282, Mar. 1995.

[2] K. Agarwal, R. Jegadeesan, Y.-X. Guo, and N. V Thakor, 'Wireless Power Transfer Strategies for Implantable Bioelectronics', IEEE Rev. Biomed. Eng., vol. 10, pp. 136-161, 2017.

[3] A. Denisov and E. Yeatman, 'Ultrasonic vs. Inductive Power Delivery for Miniature Biomedical Implants', in 2010 International Conference on Body Sensor Networks, 2010, pp. 84-89.

[4] P. Si, A. P. Hu, S. Malpas, and D. Budgett, 'A Frequency Control Method for Regulating Wireless Power to Implantable Devices', IEEE Trans. Biomed. Circuits Syst., vol. 2, no. 1, pp. 22-29, Mar. 2008.

[5] Guoxing Wang, Wentai Liu, M. Sivaprakasam, and G. A. Kendir, 'Design and analysis of an adaptive transcutaneous power telemetry for biomedical implants', IEEE Trans. Circuits Syst. I Regul. Pap., vol. 52, no. 10, pp. 2109-2117, Oct. 2005.

[6] D. Pivonka, A. Yakovlev, A. S. Y. Poon, and T. Meng, 'A mmSized Wirelessly Powered and Remotely Controlled Locomotive Implant', IEEE Trans. Biomed. Circuits Syst., vol. 6, no. 6, pp. 523532, Dec. 2012.

[7] D. Ahn and M. Ghovanloo, 'Optimal Design of Wireless Power Transmission Links for Millimeter-Sized Biomedical Implants', IEEE Trans. Biomed. Circuits Syst., vol. 10, no. 1, pp. 125-137, Feb. 2016.

[8] J. Charthad, M. J. Weber, T. C. Chang, and A. Arbabian, 'A mmSized Implantable Medical Device (IMD) With Ultrasonic Power Transfer and a Hybrid Bi-Directional Data Link', IEEE J. SolidState Circuits, vol. 50, no. 8, pp. 1741-1753, Aug. 2015.

[9] V. Y. Reddy et al., 'Cardiac Resynchronization Therapy With Wireless Left Ventricular Endocardial Pacing: The SELECT-LV Study', J. Am. Coll. Cardiol., vol. 69, no. 17, pp. 2119-2129, May 2017.

[10] R. Jegadeesan, K. Agarwal, Y.-X. Guo, S.-C. Yen, and N. V Thakor, 'Wireless Power Delivery to Flexible Subcutaneous Implants Using Capacitive Coupling', IEEE Trans. Microw. Theory Tech., vol. 65, no. 1, pp. 280-292, Jan. 2017.

[11] R. Erfani, F. Marefat, A. M. Sodagar, and P. Mohseni, 'Modeling and Experimental Validation of a Capacitive Link for Wireless Power Transfer to Biomedical Implants', IEEE Trans. Circuits Syst. II Express Briefs, vol. 65, no. 7, pp. 923-927, Jul. 2018.

[12] P. Chen, H. Yang, R. Luo, and B. Zhao, 'A Tissue-Channel Transcutaneous Power Transfer Technique for Implantable Devices', IEEE Trans. Power Electron., vol. 33, no. 11, pp. 97539761, Nov. 2018. 


\section{IEEE POWER ELECTRONICS REGULAR PAPER/LETTER/CORRESPONDENCE}

[13] Q. Castellví, Antoni Ivorra, Laura Becerra-Fajardo, 'In vivo demonstration of injectable microstimulators based on chargebalanced rectification of epidermically applied currents', J. Neural Eng., vol. 12, no. 6, p. 66010, Oct. 2015.

[14] L. Becerra-Fajardo, M. Schmidbauer, and A. Ivorra, 'Demonstration of $2 \mathrm{~mm}$ thick microcontrolled injectable stimulators based on rectification of high frequency current bursts', IEEE Trans. Neural Syst. Rehabil. Eng., vol. 25, no. 8, pp. 1343-1352, Aug. 2017.

[15] M. Tudela-Pi, L. Becerra-Fajardo, A. Garcia-Moreno, J. Minguillon, and A. Ivorra, 'Power Transfer by Volume Conduction: In Vitro Validated Analytical Models Predict DC Powers Above $1 \mathrm{~mW}$ in Injectable Implants', IEEE Access, vol. 8, pp. 37808-37820, 2020.

[16] Y. Sun and X. B. Yu, 'Capacitive Biopotential Measurement for Electrophysiological Signal Acquisition: A Review’, IEEE Sens. J., vol. 16, no. 9, pp. 2832-2853, May 2016.

[17] K. J. Chen, J. Oswald, and T. Krüger, 'Electrophoretic deposition of dielectric film on stimulation electrodes for the use in intraoperative neuromonitoring', Curr. Dir. Biomed. Eng., vol. 4, no. 1, pp. 521524, Sep. 2018.

[18] P. Single and J. Scott, 'Cause of Pulse Artefacts Inherent to the Electrodes of Neuromodulation Implants', IEEE Trans. Neural Syst. Rehabil. Eng., vol. 26, no. 10, pp. 2078-2083, Oct. 2018.

[19] J. B. Firszt, D. B. Koch, M. Downing, and L. Litvak, 'Current Steering Creates Additional Pitch Percepts in Adult Cochlear Implant Recipients', Otol. Neurotol., vol. 28, no. 5, pp. 629-636, Aug. 2007.

[20] M. Catrysse, B. Hermans, and R. Puers, 'An inductive power system with integrated bi-directional data-transmission’, Sensors Actuators A Phys., vol. 115, no. 2-3, pp. 221-229, Sep. 2004.

[21] L. Becerra-Fajardo, M. Tudela-Pi, and A. Ivorra, 'Two-Port Networks to Model Galvanic Coupling for Intrabody Communications and Power Transfer to Implants', in 2018 IEEE Biomedical Circuits and Systems Conference (BioCAS), 2018, pp. $1-4$.

[22] W. Zhang and C. C. Mi, 'Compensation Topologies of High-Power Wireless Power Transfer Systems’, IEEE Trans. Veh. Technol., vol. 65, no. 6, pp. 4768-4778, Jun. 2016.

[23] B. Lenaerts and R. Puers, Omnidirectional inductive powering for biomedical implants. Springer, 2009.

[24] R. Sedehi, D. Budgett, A. P. Hu, and D. McCormick, 'Effects of Conductive Tissue on Capacitive Wireless Power Transfer', in 2018 IEEE PELS Workshop on Emerging Technologies: Wireless Power Transfer (Wow), 2018, pp. 1-5.

[25] A. Stogryn, 'Equations for Calculating the Dielectric Constant of Saline Water (Correspondence)', IEEE Trans. Microw. Theory Tech., vol. 19, no. 8, pp. 733-736, Aug. 1971.

[26] H. R. Gamba and D. T. Delpy, 'Measurement of electrical current density distribution within the tissues of the head by magnetic resonance imaging', Med. Biol. Eng. Comput., vol. 36, no. 2, pp. 165-170, Mar. 1998.

[27] S. Gabriel, R. W. Lau, and C. Gabriel, 'The dielectric properties of biological tissues: II. Measurements in the frequency range $10 \mathrm{~Hz}$ to 20 GHz', Phys. Med. Biol., vol. 41, no. 11, pp. 2251-2269, Nov. 1996.

[28] S. Kuppusami and R. H. Oskouei, 'Parylene coatings in medical devices and implants: A review’, Univ. J. Biomed. Eng, vol. 3, no. 3, pp. 9-14, 2015.

[29] 'IEEE Standard for Safety Levels with Respect to Human Exposure to Radio Frequency Electromagnetic Fields, $3 \mathrm{kHz}$ to $300 \mathrm{GHz}$, IEEE Std C95.1-2005 (Revision of IEEE Std C95.1-1991). pp. 1238, 2006.

[30] The COMSOL Group, 'Specific Absorption Rate (SAR) and Temprature Study in Human Brain’, COMSOL. [Online]. Available: https://www.comsol.com/model/specific-absorption-rate-sar-andtemperature-study-in-the-human-brain-2190. [Accessed: 20-Jul2019].

[31] K. Bocan and E. Sejdić, 'Adaptive Transcutaneous Power Transfer to Implantable Devices: A State of the Art Review', Sensors, vol. 16, no. 3, p. 393, Mar. 2016.

[32] J. M. Rivas, Y. Han, O. Leitermann, A. D. Sagneri, and D. J. Perreault, 'A High-Frequency Resonant Inverter Topology With Low-Voltage Stress', IEEE Trans. Power Electron., vol. 23, no. 4, pp. 1759-1771, Jul. 2008.

[33] C95.1-2019 - IEEE Standard for Safety Levels with Respect to Human Exposure to Electric, Magnetic, and Electromagnetic Fields, $0 \mathrm{~Hz}$ to $300 \mathrm{GHz}$. IEEE.

[34] B. Lenaerts and R. Puers, 'An inductive power link for a wireless endoscope’, Biosens. Bioelectron., vol. 22, no. 7, pp. 1390-1395, Feb. 2007.

[35] M. Meng and M. Kiani, 'A Hybrid Inductive-Ultrasonic Link for Wireless Power Transmission to Millimeter-Sized Biomedical Implants', IEEE Trans. Circuits Syst. II Express Briefs, vol. 64, no. 10, pp. 1137-1141, Oct. 2017.

[36] D. Seo, J. M. Carmena, J. M. Rabaey, E. Alon, and M. M. Maharbiz, 'Neural Dust: An Ultrasonic, Low Power Solution for Chronic Brain-Machine Interfaces’, arXiv Prepr. arXiv1307.2196, Jul. 2013.

[37] S. L. C. Au, F.-Y. B. Chen, D. Budgett, S. C. Malpas, S.-J. Guild, and D. McCormick, 'Injection Molded Liquid Crystal Polymer Package for Chronic Active Implantable Devices with Application to an Optogenetic Stimulator', IEEE Trans. Biomed. Eng., pp. 1-1, Aug. 2019.

[38] P. E. K. Donaldson and E. Sayer, 'A technology for implantable hermetic packages. Part 2: An implementation’, Med. Biol. Eng. Comput., vol. 19, no. 4, pp. 403-405, Jul. 1981.

[39] R. Jegadeesan, S. Nag, K. Agarwal, N. V Thakor, and Y.-X. Guo, 'Enabling Wireless Powering and Telemetry for Peripheral Nerve Implants', IEEE J. Biomed. Heal. Informatics, vol. 19, no. 3, pp. 958-970, May 2015.

[40] A. Yakovlev, S. Kim, and A. Poon, 'Implantable biomedical devices: Wireless powering and communication', IEEE Commun. Mag., vol. 50, no. 4, pp. 152-159, Apr. 2012.

[41] S. M. Asif et al., 'Design and In Vivo Test of a Batteryless and Fully Wireless Implantable Asynchronous Pacing System', IEEE Trans. Biomed. Eng., vol. 63, no. 5, pp. 1070-1081, May 2016.

[42] S. A. Mirbozorgi, P. Yeon, and M. Ghovanloo, 'Robust Wireless Power Transmission to mm-Sized Free-Floating Distributed Implants', IEEE Trans. Biomed. Circuits Syst., vol. 11, no. 3, pp. 692-702, Jun. 2017.

[43] G. Wang, W. Liu, M. Sivaprakasam, M. Zhou, J. D. Weiland, and M. S. Humayun, 'A Dual Band Wireless Power and Data Telemetry for Retinal Prosthesis', in 2006 International Conference of the IEEE Engineering in Medicine and Biology Society, 2006, pp. 4392-4395.

[44] L. Huang, A. P. Hu, A. K. Swain, and Y. Su, 'Z-Impedance Compensation for Wireless Power Transfer Based on Electric Field', IEEE Trans. Power Electron., vol. 31, no. 11, pp. 75567563, Nov. 2016.

[45] T. J. Oxley et al., 'Minimally invasive endovascular stent-electrode array for high-fidelity, chronic recordings of cortical neural activity’, Nat. Biotechnol., vol. 34, no. 3, pp. 320-327, Mar. 2016. 